\title{
Stochastic independence with respect to upper and lower conditional probabilities defined by Hausdorff outer and inner measures
}

\author{
Serena Doria \\ University G.d'Annunzio \\ Italy
}

\section{Introduction}

A new model of coherent upper conditional prevision is proposed in a metric space. It is defined by the Choquet integral with respect to the s-dimensional Hausdorff outer measure if the conditioning event has positive and finite Hausdorff outer measure in its dimension $s$. Otherwise if the conditioning event has Hausdorff outer measure in its dimension equal to zero or infinity it is defined by a 0-1 valued finitely, but not countably, additive probability.

If the conditioning event has positive and finite Hausdorff outer measure in its dimension the coherent upper conditional prevision is proven to be monotone, comonotonically additive, submodular and continuous from below.

Given a coherent upper conditional prevision the coherent lower conditional prevision is defined as its conjugate.

In Doria (2007) coherent upper and lower conditional probablities are obtained when only 0-1 valued random variables are considered.

The aim of this chapter is to introduce a new definition of stochastic independence with respect to coherent upper and lower conditional probabilities defined by Hausdorff outer and inner measures.

A concept related to the definition of conditional probability is stochastic independence. In a continuous probability space where probability is usually assumed equal to the Lebesgue measure, we have that finite, countable and fractal sets (i.e. the sets with non-integer Hausdorff dimension) have probability equal to zero. For these sets the standard definition of independence given by the factorization property is always satisfied since both members of the equality are zero.

The notion of s-independence with respect to Hausdorff outer and inner measures is introduced to check probabilistic dependence for sets with probability equal to zero, which are always independent according to the standard definition given by the factorization property. Moreover s-independence is compared with the notion of epistemic independence with respect to upper and lower conditional probabilities (Walley, 1991).

The outline of the chapter is the following.

In Section 2 it is proven that a conditional prevision defined by the Radon-Nikodym derivative may be not coherent and examples are given. 
In Section 3 coherent upper conditional previsions are defined in a metric space by the Choquet integral with respect to Hausdorff outer measure if the conditioning event has positive and finite Hausdorff outer measure in its dimension. Otherwise they are defined by a 0-1 valued finitely, but not countably, additive probability. Their properties are proven.

In Section 4 the notion of s-irrelevance and s-independence with respect to coherent upper and lower conditional probabilities defined by Hausdorff outer and inner measures are introduced. It is proven that the notions of epistemic irrelevance and s-irrelevance are not always related. In particular we give conditions for which an event $B$ is epistemically irrelevant to an event $A$, but it is not s-irrelevant. In the Euclidean metric space it is proven that a necessary condition for s-irrelevance between events is that the Hausdorff dimension of the two events and their intersection is equal to the Hausdorff dimension of $\Omega$. Finally sufficient conditions for s-irrelevance between Souslin subsets of $\Re^{n}$ are given.

In Section 5 some fractal sets are proven to be s-dependent since they do not satisfy the necessary condition for s-independence. In particular the attractor of a finite family of similitudes and its boundary are proven to be s-dependent if the open set condition holds. Moreover a condition for which two middle Cantor sets are s-dependent is given.

It is important to note that all these sets are stochastically independent according the axiomatic definition given by the factorization property if probability is defined by the Lebesgue measure.

In Section 6 curves filling the space, such as Peano curve and Hilbert curve are proven to be s-independent.

\section{Conditional expectation and coherent conditional prevision}

Partial knowledge is a natural interpretation of conditional probability. This interpretation can be formalized in a different way in the axiomatic approach and in the subjective approach where conditional probability is respectively defined by the Radon-Nikodym derivative or by the axioms of coherence. In both cases conditional probability is obtained as the restriction of conditional expectation or conditional prevision to the class of indicator functions of events. Some critical situations, which highlight as the axiomatic definition of conditional probability is not always a useful tool to represent partial knoweledge, are proposed in literature and analyzed in this section. In particular the role of the Radon-Nikodym derivative in the assessment of a coherent conditional prevision is investigated.

It is proven that, every time that the $\sigma$-field of the conditioning events is properly contained in the $\sigma$-field of the probability space and it contains all singletons, the Radon-Nikodym derivative cannot be used as a tool to define coherent conditional previsions. This is due to the fact that one of the defining properties of the Radon-Nikodym derivative, that is to be measurable with respect to the $\sigma$-field of the conditioning events, contradicts a necessary condition for the coherence.

Analysis done points out the necessity to introduce a different tool to define coherent conditional previsions.

\subsection{Conditional expectation and Radon-Nikodym derivative}

In the axiomatic approach Billingsley (1986) conditional expectation is defined with respect to a $\sigma$-field $\mathbf{G}$ of conditioning events by the Radon-Nikodym derivative. Let $(\Omega, \mathbf{F}, P)$ be a probability space and let $\mathbf{F}$ and $\mathbf{G}$ be two $\sigma$-fields of subsets of $\Omega$ with $\mathbf{G}$ contained in $\mathbf{F}$ and let $X$ be an integrable random variable on $(\Omega, \mathbf{F}, P)$. Let $P$ be a probability measure on $\mathbf{F}$; define a measure $v$ on $\mathbf{G}$ by $v(G)=\int_{G} X d P$. This measure is finite and absolutely continuous with 
respect to $P$. So there exists a function, the Radon-Nikodym derivative denoted by $E[X \mid \mathbf{G}]$, defined on $\Omega$, G-measurable, integrable and satisfying the functional equation

$$
\int_{G} E[X \mid \mathbf{G}] d P=\int_{G} X d P \text { with } G \text { in } \mathbf{G} .
$$

This function is unique up to a set of $P$-measure zero and it is a version of the conditional expected value.

If $X$ is the indicator function of any event $A$ belonging to $\mathbf{F}$ then $E[X \mid \mathbf{G}]=E[A \mid \mathbf{G}]=P[A \mid \mathbf{G}]$ is a version of the conditional probability.

Conditional probability can be used to represent partial information (Billingsley, 1986, Section 33).

A probability space $(\Omega, \mathbf{F}, P)$ can be use to represent a random phenomenon or an experiment whose outcome is drawn from according to the probability given by $P$. Partial information about the experiment can be represented by a sub $\sigma$-field $\mathbf{G}$ of $\mathbf{F}$ in the following way: an observer does not know which $\omega$ has been drawn but he knows for each $H \in \mathbf{G}$, if $\omega$ belongs to $H$ or if $\omega$ belongs to $H^{c}$. A sub $\sigma$-field $\mathbf{G}$ of $\mathbf{F}$ can be identified as partial information about the random experiment, and, fixed $A$ in $\mathbf{F}$, conditional probability can be used to represent partial knowledge about $A$ given the information on G. If conditional probability is defined by the Radon-Nykodim derivative, denoted by $P[A \mid \mathbf{G}]$, by the standard definition (Billingsley, 1986, p.52) we have that an event $A$ is independent from the $\sigma$-field $\mathbf{G}$ if it is independent from each $H \in \mathbf{G}$, that is $P[A \mid \mathbf{G}]=P(A)$ with probability 1. In (Billingsley, 1986, Example 33.11) it is shown that the interpretation of conditional probability in terms of partial knowledge breaks down in certain cases. Let $\Omega=[0,1]$, let $\mathbf{F}$ be the Borel $\sigma$-field of $[0,1]$ and let $P$ be the Lebesgue measure on F. Let $\mathbf{G}$ be the sub $\sigma$-field of sets that are either countable or co-countable. Then $P(A)$ is a version of the conditional probability $P[A \mid \mathbf{G}]$ define by the Radon-Nikodym derivative because $P(G)$ is either 0 or 1 for evey $G \in \mathbf{G}$. So an event $A$ is independent from the information represented by $\mathbf{G}$ and this is a contradiction according to the fact that the information represented by $\mathbf{G}$ is complete since $\mathbf{G}$ contains all the singletons of $\Omega$.

\subsection{Coherent upper conditional previsions}

In the subjective probabilistic approach (de Finetti 1970, Dubins 1975 and Walley 1991) coherent upper conditional previsions $\bar{P}(\cdot \mid B)$ are functionals, defined on a linear space of bounded random variables, satisfying the axioms of coherence.

In Walley (1991) coherent upper conditional previsions are defined when the conditioning events are sets of a partition.

Definition 1. Let $\Omega$ be a non-empty set let $\boldsymbol{B}$ be a partition of $\Omega$. For every $B \in \boldsymbol{B}$ let $\boldsymbol{K}(B)$ be a linear space of bounded random variables defined on $B$. Then separately coherent upper conditional previsions are functionals $\bar{P}(\cdot \mid B)$ defined on $K(B)$, such that the following conditions hold for every $X$ and $Y$ in $K(B)$ and every strictly positive constant $\lambda$ :

- 1) $\bar{P}(X \mid B) \leq \sup (X \mid B)$;

- 2) $\bar{P}(\lambda X \mid B)=\lambda \bar{P}(X \mid B)$ (positive homogeneity);

- 3) $\bar{P}(X+Y) \mid B) \leq \bar{P}(X \mid B)+\bar{P}(Y \mid B)$;

- 4) $\bar{P}(B \mid B)=1$. 
Coherent conditional upper previsions can always be extended to coherent upper previsions on the class $\mathbf{L}(B)$ of all bounded random variables defined on $B$.

Suppose that $\bar{P}(X \mid B)$ is a coherent upper conditional prevision on $\mathbf{K}$ then its conjugate coherent lower conditional prevision is defined by $\underline{P}(-X \mid B)=-\bar{P}(X \mid B)$. If for every $X$ belonging to $\mathbf{K}$ we have $P(X \mid B)=\underline{P}(X \mid B)=\bar{P}(X \mid B)$ then $P(X \mid B)$ is called a coherent linear conditional prevision de Finetti (1970) and it is a linear positive functional on $\mathbf{K}$.

Definition 2. Let $\Omega$ be a non-empty set let $\boldsymbol{B}$ be a partition of $\Omega$. For every $B \in \boldsymbol{B}$ let $\boldsymbol{K}(B)$ be a linear space of bounded random variables defined on $B$. Then linear coherent conditional previsions are functionals $P(\cdot \mid B)$ defined on $K(B)$, such that the following conditions hold for every $X$ and $Y$ in $K(B)$ and every strictly positive constant $\lambda$ :

- 1) if $X>0$ then $P(X \mid B) \geq 0$ (positivity);

- 2) $P(\lambda X \mid B)=\lambda P(X \mid B)$ (positive homogeneity);

- 3) $P(X+Y) \mid B)=P(X \mid B)+P(Y \mid B)$ (linearity);

- 4) $P(B \mid B)=1$.

Upper conditional probabilities are obtained when only 0-1 valued random variables are considered;

In Dubins (1975) coherent conditional probabilities are defined when the family of the conditioning events is a field of subsets of $\Omega$.

Definition 3. Let $\Omega$ be a non-empty set and let $\boldsymbol{F}$ and $\boldsymbol{G}$ be two fields of subsets of $\Omega$, with $G \subseteq \boldsymbol{F}$. $P$ is a finitely additive conditional probability on $(\boldsymbol{F}, \boldsymbol{G})$ if it is a real function defined on $\boldsymbol{F} \times \boldsymbol{G}^{0}$, where $G^{0}=G-\oslash$ such that the following conditions hold:

- I) given any $H \in G^{0}$ and $A_{1}, \ldots, A_{n} \in \boldsymbol{F}$ and $A_{i} \cap A_{j}=\oslash$ for $i \neq j$, the function $P(\cdot \mid H)$ defined on $\boldsymbol{F}$ is such that $P(A \mid H) \geq 0, P\left(\bigcup_{k=1}^{n} A_{k} \mid H\right)=\sum_{k=1}^{n} P\left(A_{k} \mid H\right), P(\Omega \mid H)=1$

- II) $P(H \mid H)=1$ if $H \in G^{0}$

- III) given $E \in \boldsymbol{F}, H \in \boldsymbol{F}$ with $A \in G^{0}$ and $E A \in G^{0}$ then $P(E H \mid A)=P(E \mid A) P(H \mid E A)$.

From conditions I) and II) we have

II') $P(A \mid H)=1$ if $A \in \mathbf{F}, H \in \mathbf{G}^{0}$ and $H \subset A$.

These conditional probabilities are coherent in the sense of de Finetti, since conditions I), II), III) are sufficient for the coherence of $P$ on $\mathbf{C}=\mathbf{F} \times \mathbf{G}^{0}$ when $\mathbf{F}$ and $\mathbf{G}$ are fields of subsets of $\Omega$ with $\mathbf{G} \subseteq \mathbf{F}$ or $\mathbf{G}$ is an additive subclass of $\mathbf{F}$; otherwise if $\mathbf{F}$ and $\mathbf{G}$ are two arbitrary families of subsets of $\Omega$, such that $\Omega \in \mathbf{F}$ the previous conditions are necessary for the coherence but not sufficient.

\subsection{Coherent conditional previsions and the Radon-Nikodym derivative}

In this subsection the role of the Radon-Nikodym derivative in the assessment of a coherent conditional prevision is analyzed.

The definitions of conditional expectation and coherent linear conditional prevision can be compared when the $\sigma$-field $\mathbf{G}$ is generated by the partition $\mathbf{B}$. Let $\mathbf{G}$ be equal or contained in the $\sigma$-field generated by a countable class $\mathbf{C}$ of subsets of $\mathbf{F}$ and let $\mathbf{B}$ be the partition generated by the class $\mathbf{C}$. Denote $\Omega^{\prime}=\mathbf{B}$ and $\varphi_{B}$ the function from $\Omega$ to $\Omega^{\prime}$ that associates to every $\omega \in \Omega$ the atom $B$ of the partition $\mathbf{B}$ that contains $\omega$; then we have that $P(\mathbf{A} \mid \mathbf{G})=P(\mathbf{A} \mid \mathbf{B}) \circ \varphi_{B}$ for every $A \in \mathbf{F}$ (Koch, 1997, 262). 
The next theorem shows that every time that the $\sigma$-field $\mathbf{G}$ of the conditioning events is properly contained in $\mathbf{F}$ and it contains all singletons of $[0,1]$ then the conditional prevision, defined by the Radon-Nikodym derivative is not coherent. It occurs because one of the defining properties of conditional expectation that is to be measurable with respect to the $\sigma$-field of conditioning events contradicts a necessary condition for coherence of a linear conditional prevision. A bounded random variable is called $\mathbf{B}$-measurable or measurable with respect to the partition $\mathbf{B}$ (Walley, 1991, p.291) if it is constant on the atoms $B$ of the partition. If for every $B$ belonging to $\mathbf{B} P(X \mid B)$ are coherent linear conditional previsions and $X$ is $\mathbf{B}$-measurable then $P(X \mid B)=X$ (Walley, 1991, p.292). This necessary condition for coherence is not always satisfied if $P(X \mid B)$ is defined by the Radon-Nikodym derivative.

Theorem 1. Let $\Omega=[0,1]$, let $\boldsymbol{F}$ be the Borel $\sigma$-field of $[0,1]$ and let $P$ be the Lebesgue measure on $\boldsymbol{F}$. Let $\boldsymbol{G}$ be a sub $\sigma$-field properly contained in $\boldsymbol{F}$ and containing all singletons of $[0,1]$. Let $\boldsymbol{B}$ be the partition of all singletons of $[0,1]$ and let $X$ be the indicator function of an event $A$ belonging to $\boldsymbol{F}-\boldsymbol{G}$. If we define the conditional prevision $P(X \mid\{\omega\})$ equal to the Radon-Nikodym derivative with probability 1 , that is

$$
P(X \mid\{\omega\})=E[X \mid G]
$$

except on a subset $N$ of $[0,1]$ of P-measure zero, then the conditional prevision $P(X \mid\{\omega\})$ is not coherent.

Proof. If the equality $P(X \mid\{\omega\})=E[X \mid \mathbf{G}]$ holds with probability 1 , then we have that, with probability 1 , the linear conditional prevision $P(X \mid\{\omega\})$ is different from $X$, the indicator function of $A$; in fact having fixed $A$ in $\mathbf{F}-\mathbf{G}$ the indicator function $X$ is not $\mathbf{G}$-measurable, it does not verify a property of the Radon-Nikodym derivative and therefore it cannot be assumed as conditional expectation according to axiomatic definition. So the linear conditional prevision $P(X \mid\{\omega\})$ does not satisfy the necessary condition for being coherent, $P(X \mid\{\omega\})=X$ for every singleton $\{\omega\}$ of $\mathbf{G} . \diamond$

Example 1. (Billingsley, 1986, Example 33.11) Let $\Omega=[0,1]$, let $\mathbf{F}$ be the Borel $\sigma$-field of $\Omega$, let $P$ be the Lebesgue measure on $\mathbf{F}$ and let $\mathbf{G}$ be the sub $\sigma$-field of $\mathbf{F}$ of sets that are either countable or co-countable. Let $\mathbf{B}$ be the partition of all singletons of $\Omega$; if the linear conditional prevision is defined equal, with probability 1 , to conditional expectation defined by the Radon-Nikodym derivative, we have that

$$
P(X \mid \mathbf{B})=E[X \mid \mathbf{G}]=P(X) .
$$

So when $X$ is the indicator function of an event $A=[a, b]$ with $0<a<b<1$ then $P(X \mid B)=$ $P(A)$ and it does not satisfy the necessary condition for coherence that is $P(X \mid\{\omega\})=X$ for every singleton $\{\omega\}$ of $\mathbf{G}$.

Evident from Theorem 1 and Example 1 is the necessity to introduce a new tool to define coherent linear conditional previsions.

\section{Coherent upper conditional previsions defined by Hausdorff outer measures}

In this section coherent upper conditional previsions are defined by the Choquet integral with respect to Hausdorff outer measures if the conditioning event $B$ has positive and finite Hausdorff outer measure in its dimension. Otherwise if the conditioning event $B$ has Hausdorff outer measure in its dimension equal to zero or infinity they are defined by a $0-1$ valued finitely, but not countably, additive probability. 


\subsection{Hausdorff outer measures}

Given a non-empty set $\Omega$ an outer measure is a function $\mu^{*}: \wp(\Omega) \rightarrow[0,+\infty]$ such that $\mu^{*}(\oslash)=0, \mu^{*}(A) \leq \mu^{*}\left(A^{\prime}\right)$ if $A \subseteq A^{\prime}$ and $\mu^{*}\left(\bigcup_{i=1}^{\infty} A_{i}\right) \leq \sum_{i=1}^{\infty} \mu^{*}\left(A_{i}\right)$.

Examples of outer set functions or outer measures are the Hausdorff outer measures (Falconer 1986, Rogers 1998).

Let $(\Omega, d)$ be a metric space. A topology, called the metric topology, can be introduced into any metric space by defining the open sets of the space as the sets $G$ with the property:

if $x$ is a point of $G$, then for some $r>0$ all points y with $d(x, y)<r$ also belong to $G$.

It is easy to verify that the open sets defined in this way satisfy the standard axioms of the system of open sets belonging to a topology (Rogers, 1998, p.26).

The diameter of a non empty set $U$ of $\Omega$ is defined as $|U|=\sup \{d(x, y): x, y \in U\}$ and if a subset $A$ of $\Omega$ is such that $A \subset U_{i} U_{i}$ and $0<\left|U_{i}\right|<\delta$ for each $\mathrm{i}$, the class $\left\{U_{i}\right\}$ is called a $\delta$-cover of $A$.

Let $\mathrm{s}$ be a non-negative number. For $\delta>0$ we define $h_{s, \delta}(A)=\inf \sum_{i=1}^{+\infty}\left|U_{i}\right|^{s}$, where the infimum is over all $\delta$-covers $\left\{U_{i}\right\}$.

The Hausdorff s-dimensional outer measure of $A$, denoted by $h^{\mathcal{S}}(A)$, is defined as

$$
h^{S}(A)=\lim _{\delta \rightarrow 0} h_{s, \delta}(A) .
$$

This limit exists, but may be infinite, since $h_{s, \delta}(A)$ increases as $\delta$ decreases because less $\delta$ covers are available. The Hausdorff dimension of a set $A$, $\operatorname{dim}_{H}(A)$, is defined as the unique value, such that

$$
\begin{aligned}
& h^{s}(A)=+\infty \text { if } 0 \leq s<\operatorname{dim}_{H}(A) \\
& h^{s}(A)=0 \text { if } \operatorname{dim}_{H}(A)<s<+\infty .
\end{aligned}
$$

We can observe that if $0<h^{s}(A)<+\infty$ then $\operatorname{dim}_{H}(A)=s$, but the converse is not true.

Denote by $r$ the Hausdorff dimension of $\Omega$, if an event $A$ is such that $\operatorname{dim}_{H}(A)=s<r$ then the Hausdorff dimension of the complementary set $A^{c}$ is equal to $r$ since the following relation holds:

$$
\operatorname{dim}_{H}(A \cup B)=\max \left\{\operatorname{dim}_{H}(A), \operatorname{dim}_{H}(B)\right\} .
$$

Hausdorff outer measures are metric outer measures, that is $h^{S}(E \cup F)=h^{S}(E)+h^{\mathcal{S}}(F)$ whenever $E$ and $F$ are positively separated, i.e. $d(E, F)=\inf \{d(x, y): x \in E, y \in F\}>0$.

A subset $A$ of $\Omega$ is called measurable with respect to the outer measure $h^{s}$ if it decomposes every subset of $\Omega$ additively, that is if $h^{s}(E)=h^{s}(A \cap E)+h^{s}(E-A)$ for all sets $E \subseteq \Omega$.

All Borel subsets of $\Omega$ are measurable with respect to a metric outer measure (Falconer, 1986, Theorem 1.5). So every Borel subset of $\Omega$ is measurable with respect to every Hausdorff outer measure $h^{s}$ since Hausdorff outer measures are metric.

The restriction of $h^{s}$ to the $\sigma$-field of $h^{s}$-measurable sets, containing the $\sigma$-field of the Borel sets, is called Hausdorff $s$-dimensional measure. The Borel $\sigma$-field is the $\sigma$-field generated by all open sets. The Borel sets include the closed sets (as complement of the open sets), the $F_{\sigma}$-sets (countable unions of closed sets) the $G_{\sigma}$-sets (countable intersections of open sets), etc. In particular the Hausdorff 0 -dimensional measure is the counting measure and the Hausdorff 1-dimensional measure is the Lebesgue measure.

The Hausdorff s-dimensional measures are modular on the Borel $\sigma$-field, that is $h^{\mathrm{s}}(A \cup B)+$ $h^{\mathcal{S}}(A \cap B)=h^{\mathcal{S}}(A)+h^{\mathcal{S}}(B)$ for every pair of Borelian sets $A$ and $B$; so that (Denneberg, 
1994, Proposition 2.4) the Hausdorff outer measures are submodular $\left(h^{s}(A \cup B)+h^{s}(A \cap B) \leq\right.$ $\left.h^{S}(A)+h^{s}(B)\right)$.

In (Rogers, 1998, p.50) and (Falconer, 1986, Theorem 1.6 (a)) it has been proven that if $A$ is any subset of $\Omega$ there is a $G_{\sigma}$-set $G$ containing $A$ with $h^{s}(A)=h^{s}(G)$. In particular $h^{s}$ is an outer regular measure.

Moreover Hausdorff outer measures are continuous from below (Falconer, 1986, Lemma 1.3), that is for any increasing sequences of sets $\left\{A_{i}\right\}$ we have $\lim _{i \rightarrow \infty} h^{\mathcal{s}}\left(A_{i}\right)=h^{\mathcal{s}}\left(\lim _{i \rightarrow \infty} A_{i}\right)$.

\subsection{The Choquet integral}

We recall the definition of the Choquet integral (Denneberg, 1994) with the aim to define upper conditional previsions by Choquet integral with respect to Hausdorff outer measures and to prove their properties. The Choquet integral is an integral with respect to a monotone set function. Given a non-empty set $\Omega$ and denoted by $S$ a set system, containing the empty set and properly contained in $\wp(\Omega)$, the family of all subsets of $\Omega$, a monotone set function $\mu$ : $S \rightarrow \bar{\Re}_{+}=\Re_{+} \cup\{+\infty\}$ is such that $\mu(\oslash)=0$ and if $A, B \in S$ with $A \subseteq B$ then $\mu(A) \leq \mu(B)$. Given a monotone set function $\mu$ on $S$, its outer set function is the set function $\mu^{*}$ defined on the whole power set $\wp(\Omega)$ by

$$
\mu^{*}(A)=\inf \{\mu(B): B \supset A ; B \in S\}, A \in \wp(\Omega)
$$

The inner set function of $\mu$ is the set function $\mu_{*}$ defined on the whole power set $\wp(\Omega)$ by

$$
\mu_{*}(A)=\sup \{\mu(B) \mid B \subset A ; B \in S\}, A \in \wp(\Omega)
$$

Let $\mu$ be a monotone set function defined on $S$ properly contained in $\wp(\Omega)$ and $X: \Omega \rightarrow \bar{\Re}=$ $\Re \cup\{-\infty,+\infty\}$ an arbitrary function on $\Omega$. Then the set function

$$
G_{\mu, X}(x)=\mu\{\omega \in \Omega: X(\omega)>x\}
$$

is decreasing and it is called decreasing distribution function of $X$ with respect to $\mu$. If $\mu$ is continuous from below then $G_{\mu, X}(x)$ is right continuous. In particular the decreasing distribution function of $X$ with respect to the Hausdorff outer measures is right continuous since these outer measures are continuous from below. A function $X: \Omega \rightarrow \bar{\Re}$ is called upper $\mu$ measurable if $G_{\mu^{*}, X}(x)=G_{\mu_{*}, X}(x)$. Given an upper $\mu$-measurable function $X: \Omega \rightarrow \bar{R}$ with decreasing distribution function $G_{\mu, X}(x)$, if $\mu(\Omega)<+\infty$, the asymmetric Choquet integral of $X$ with respect to $\mu$ is defined by

$$
\int X d \mu=\int_{-\infty}^{0}\left(G_{\mu, X}(x)-\mu(\Omega)\right) d x+\int_{0}^{\infty} G_{\mu, X}(x) d x
$$

The integral is in $\Re$, can assume the values $-\infty,+\infty$ or is undefined when the right-hand side is $\infty-\infty$.

If $X \geq 0$ or $X \leq 0$ the integral always exists. In particular for $X \geq 0$ we obtain

$$
\int X d \mu=\int_{0}^{+\infty} G_{\mu, X}(x) d x
$$

If $X$ is bounded and $\mu(\Omega)=1$ we have that

- $\int X d \mu=\int_{\inf X}^{0}\left(G_{\mu, X}(x)-1\right) d x+\int_{0}^{\sup X} G_{\mu, X}(x) d x=\int_{\inf X}^{\sup X} G_{\mu, X}(x) d x+\inf X$. 


\subsection{A new model of coherent upper conditional prevision}

A new model of coherent upper conditional prevision is introduced and its properties are proven.

Theorem 2. Let $(\Omega, d)$ be a metric space and let $\boldsymbol{B}$ be a partition of $\Omega$. For every $B \in \boldsymbol{B}$ denote by $s$ the Hausdorff dimension of the conditioning event $B$ and by $h^{s}$ the Hausdorff s-dimensional outer measure. Let $\mathbf{L}(B)$ be the class of all bounded random variables on $B$. Moreover, let $m$ be a 0-1 valued finitely additive, but not countably additive, probability on $\wp(B)$. Then for each $B \in \boldsymbol{B}$ the functionals $\bar{P}(X \mid B)$ defined on $\mathbf{L}(B)$ by

$$
\bar{P}(X \mid B)=\frac{1}{h^{s}(B)} \int_{B} X d h^{s} \text { if } 0<h^{s}(B)<+\infty
$$

and by

$$
\bar{P}(X \mid B)=m(X B) \text { if } h^{s}(B)=0,+\infty
$$

are coherent upper conditional previsions.

Proof. Since $\mathbf{L}(B)$ is a linear space we have to prove that, for every $B \in \mathbf{B} \bar{P}(X \mid B)$ satisfies conditions 1), 2), 3), 4) of Definition 1.

If $B$ has finite and positive Hausdorff outer measure in its dimension $s$ then $\bar{P}(X \mid B)=\frac{1}{h^{s}(B)} \int_{B} X d h^{s}$, so properties 1) and 2) are satisfied since they hold for the Choquet integral (Denneberg, 1994, Proposition 5.1). Property 3) follows from the Subadditivity Theorem (Denneberg, 1994, Theorem 6.3) since Hausdorff outer measures are monotone, submodular and continuous from below. Property 4) holds since $\bar{P}(B \mid B)=\frac{1}{h^{s}(B)} \int_{B} d h^{s}=1$. If $B$ has Hausdorff outer measure in its dimension equal to zero or infinity we have that the class of all coherent (upper) previsions on $\mathbf{L}(B)$ is equivalent to the class of $0-1$ valued additive probabilities defined on $\wp(B)$ then $\bar{P}(X \mid B)=m(X B)$. Then properties 1$), 2), 3)$ are satisfied since $m$ is a $0-1$ valued finitely additive probability on $\wp(B)$. Moreover since a different $m$ is choosen for each $B$ we have that $\bar{P}(B \mid B)=m(B)=1$.

The lower conditional previsions $\underline{P}(A \mid B)$ can be defined as the previous theorem if $h_{s}$ denotes the Hausdorff s-dimensional inner measure. The unconditional upper prevision is obtained as a particular case when the conditioning event is $\Omega$, that is $\bar{P}(A)=\bar{P}(A \mid \Omega)$ and $\underline{P}(A)=$ $\underline{P}(A \mid \Omega)$.

$\bar{A}$ class of bounded random variables is called a lattice if it is closed under point-wise maximum $\vee$ and point-wise minimum $\wedge$.

In the following theorem it is proven that, if the conditioning event has positive and finite Hausdorff outer measure in its dimension $s$ and $\mathrm{L}(\mathrm{B})$ is a linear lattice of bounded random variables defined on $B$, necessary conditions for the functional $\bar{P}(X \mid B)$ to be represented as Choquet integral with respect to the upper conditional probability $\mu_{B}^{*}$, i.e. $\bar{P}(X \mid B)=$ $\frac{1}{h^{s}(B)} \int X d h^{s}$, are that $\bar{P}(X \mid B)$ is monotone, comonotonically additive, submodular and continuous from below.

Theorem 3. Let $(\Omega, d)$ be a metric space and let $\boldsymbol{B}$ be a partition of $\Omega$. For every $B \in \boldsymbol{B}$ denote by $s$ the Hausdorff dimension of the conditioning event $B$ and by $h^{s}$ the Hausdorff s-dimensional outer measure. Let $L(B)$ be a linear lattice of bounded random variables defined on $B$. If the conditioning event $B$ has positive and finite Hausdorff s-dimensional outer measure in its dimension then the upper conditional prevision $\bar{P}(\cdot \mid B)$ defined on $\mathbf{L}(B)$ as in Theorem 2 satisfies the following properties:

- i) $X \leq Y$ implies $\bar{P}(X \mid B) \leq \bar{P}(Y \mid B)$ (monotonicity); 
- ii) if $X$ and $Y$ are comonotonic, i.e. $\left(X\left(\omega_{1}\right)-X\left(\omega_{2}\right)\right)\left(Y\left(\omega_{1}\right)-\left(Y\left(\omega_{2}\right)\right) \geq 0 \forall \omega_{1}, \omega_{2} \in B\right.$, then $\bar{P}(X+Y \mid B)=\bar{P}(X \mid B)+\bar{P}(Y \mid B)$ (comonotonic additivity);

- iii) $\bar{P}(X \vee Y \mid B)+\bar{P}(X \wedge Y \mid B) \leq \bar{P}(X \mid B)+\bar{P}(Y \mid B)$ (submodularity);

- iv) $\lim _{n \rightarrow \infty} \bar{P}\left(X_{n} \mid B\right)=\bar{P}(X \mid B)$ if $X_{n}$ is an increasing sequence of random variables converging to $X$ (continuity from below).

Proof. Since the conditioning event $B$ has positive and finite Hausdorff outer measure in its dimension $s$ then the functional $\bar{P}(\cdot \mid B)$ is defined on $\mathbf{L}(B)$ by the Choquet integral with respect to the upper conditional probability $\mu_{B}^{*}(A)=\frac{h^{s}(A B)}{h^{s}(B)}$; so conditions $i$ ) and ii) are satisfied because they are properties of the Choquet integral (Denneberg, 1994, Proposition 5.2).

Condition $\mathrm{iii}$ ) is equivalent to require that the monotone set function that represents the functional $\bar{P}(\cdot \mid B)$ is submodular and it is satisfied since Hausdorff outer measures are submodular. Moreover every s-dimensional Hausdorff measure is continuous from below then from the Monotone Convergence Theorem (Denneberg, 1994, Theorem 8.1) we have that the functional $\bar{P}(\cdot \mid B)$ is continuous from below, that is condition iv). $\diamond$

Coherent upper conditional probabilities are obtained when only 0-1 valued random variables are considered;

Theorem 4. Let $(\Omega, d)$ be a metric space and let $\boldsymbol{B}$ be a partition of $\Omega$. For every $B \in \boldsymbol{B}$ denote by s the Hausdorff dimension of the conditioning event $B$ and by $h^{s}$ the Hausdorff s-dimensional outer measure. Let $m$ be a 0-1 valued finitely additive, but not countably additive, probability on $\wp(B)$. Then, for each $B \in \boldsymbol{B}$, the functions defined on $\wp(B)$ by

$$
\bar{P}(A \mid B)=\frac{h^{s}(A B)}{h^{s}(B)} \text { if } 0<h^{s}(B)<+\infty
$$

and by

$$
\bar{P}(A \mid B)=m(A B) \text { if } h^{S}(B)=0,+\infty
$$

are coherent upper conditional probabilities.

Coherent upper conditional probabilities can be defined in the general case where the family of the conditioning events is an additive class of events; they have been defined in Doria (2007):

Theorem 5. Let $(\Omega, d)$ be a metric space, let $\boldsymbol{F}$ be the $\sigma$-field of all subsets of $\Omega$ and let $\boldsymbol{G}$ be an additive subclass of $\boldsymbol{F}$. For every $H \in G^{0}=G-\oslash$ and $A \in \boldsymbol{F}$ denote by s the Hausdorff dimension of the conditioning event $H$, by the Hausdorff dimension of $A H$ and by $h^{s}$ the Hausdorff s-dimensional outer measure. Let $m$ be a 0-1 valued finitely additive, but not countably additive, probability on $\wp(H)$ such that if $0<h^{t}(A H)<+\infty$ then $m(A H)=0$. Then, for each $H \in G^{0}$, the functions defined on $\wp(H)$ by

$$
\bar{P}(A \mid H)=\frac{h^{s}(A H)}{h^{s}(H)} \text { if } 0<h^{s}(H)<+\infty
$$

and by

$$
\bar{P}(A \mid H)=m(A H) \text { if } h^{s}(H)=0,+\infty
$$

are coherent upper conditional probabilities. 
The lower conditional probability $\underline{P}(A \mid H)$ can be defined as in the previous theorem by the Hausdorff inner measures.

The new model of upper and lower conditional probabilities defined as in Theoren 5 can be used to assess coherent upper and lower conditional probabilities when the extensions of the conditional probability, defined in the axiomatic way, are not coherent.

Example 2. Let $\Omega=[0,1]$, let $\mathbf{F}$ be the class of all subsets of $\Omega$ and let $\mathbf{G}$ be the $\sigma$-field of countable and co-countable subsets of $\Omega$. From Theorem 5 we have that a coherent upper conditional probability on $\mathbf{C}=\mathbf{F} \times \mathbf{G}^{0}$ can be defined by

$$
\begin{gathered}
\bar{P}(A \mid H)=\frac{h^{1}(A H)}{h^{1}(H)} \text { if } H \text { is co-countable } \\
\bar{P}(A \mid H)=\frac{h^{0}(A H)}{h^{0}(H)} \text { if } H \text { is finite } \\
\bar{P}(A \mid H)=m(A H) \text { if } H \text { is countable. }
\end{gathered}
$$

\section{4. s-Irrelevance and s-independence}

In a recent paper (Doria, 2007) the new definitions of s-irrelevance and s-independence with respect to upper and lower conditional probabilities assigned by outer and inner Hausdorff measures have been proposed. They are based on the fact that epistemic independence and irrelevance, introduced by Walley, must be tested for events $A$ and $B$, such that they and their intersection $A B$, have the same Hausdorff dimension. The concept of epistemic independence (Walley, 1991) is based on the notion of irrelevence; given two events $A$ and $B$, we say that $B$ is irrelevant to $A$ when $\bar{P}(A \mid B)=\bar{P}\left(A \mid B^{c}\right)=\bar{P}(A)$ and $\underline{P}(A \mid B)=\underline{P}\left(A \mid B^{c}\right)=\underline{P}(A)$.

The events $A$ and $B$ are epistemically independent when $B$ is irrelevant to $A$ and $A$ is irrelevant to $B$. As a consequence of this definition we can obtain that the factorization property $P(A B)=P(A) P(B)$, which constitutes the standard definition of independence for events, holds either for $P=\bar{P}$ and $P=\underline{P}$. In a continuous probabilistic space $(\Omega, F, P)$, where $\Omega$ is equal to $[0,1]^{n}$ and the probability is usually assumed equal to the Lebesgue measure on $\Omega$, we have that the finite, countable and fractal sets (i.e. the sets with Hausdorff dimension non integer) have probability equal to zero. For these sets the standard definition of independence, given by the factorization property, is always satisfied since both members of the equality are zero. In Theorem 6 of this Section we prove that an event $B$ is always irrelevant, according to the definition of Walley, to an event $A$ if $\operatorname{dim}_{H}(A)<\operatorname{dim}_{H}(B)<\operatorname{dim}_{H}(\Omega)$ and $A$ and $B$ have positive and finite Hausdorff outer measures in their dimensions; moreover if $A$ and $B$ are disjoint then they are epistemically independent. Nevertheless $B$ is not s-irrelevant to $A$.

To avoid these problems the notions of s-irrelevance and s-independence with respect to upper and lower conditional probabilities assigned by a class of Hausdorff outer and inner measures are proposed to test independence. The definitions of s-independence and s-irrelevance are based on the fact that epistemic independence and irrelevance, must be tested for events $A$ and $B$, such that they and their intersection $A B$, have the same Hausdorff dimension. According to this approach to independence, sets that represent events can be imagined divided in different layers; in each layer there are sets with the same Hausdorff dimension; two events $A$ and $B$ are s-independent if and only if the events $A$ and $B$ and their intersection $A B$ belong to the same layer and they are epistemically independent.

Definition 4 Let $(\Omega, d)$ be a metric space. Denote by $\mathbf{F}$ the $\sigma$-field of all subsets of $\Omega$ and by $\mathbf{G}^{0}=\mathbf{F}-\oslash$. Denoted by $\bar{P}$ and $\underline{P}$ the upper and lower conditional probabilities defined as in 
Theorem 5 and given $A$ and $B$ in $\mathbf{G}^{0}$, then they are s-independent if the following conditions hold:

- 1s) $\operatorname{dim}_{H}(A B)=\operatorname{dim}_{H}(B)=\operatorname{dim}_{H}(A)$

- 2s) $\bar{P}(A \mid B)=\bar{P}\left(A \mid B^{c}\right)=\bar{P}(A)$ and $\underline{P}(A \mid B)=\underline{P}\left(A \mid B^{c}\right)=\underline{P}(A)$;

- 3s) $\bar{P}(B \mid A)=\bar{P}\left(B \mid A^{c}\right)=\bar{P}(B)$ and $\underline{P}(B \mid A)=\underline{P}\left(B \mid A^{c}\right)=\underline{P}(B)$;

$B$ is s-irrelevant to $A$ if conditions $1 \mathrm{~s}$ ) and $2 \mathrm{~s}$ ) hold and $A$ is s-irrelevant to $B$ if conditions $1 \mathrm{~s}$ ) and 3 s) hold.

Remark 1 Two disjoint events $A$ and $B$ are s-dependent since the Hausdorff dimension of the empty set cannot be equal to that one of any other set so condition 1s) is never satisfied.

Given the Euclidean metric space $\left([0,1]^{n}, d\right)$ in Doria (2007) it is proven that logical independence is a necessary condition for $s$-independence for events with Hausdorff dimension less then $n$.

Example 3 Let $\Omega=[0,1]$ let $A$ be the Cantor set and let $B$ be a finite subset of $\Omega$ such that intersection $A B$ is equal to the empty set. We recall the definition of the Cantor set.

Let $E_{0}=[0,1], E_{1}=[0,1 / 3] \cup[2 / 3,1], E_{2}=[0,1 / 9] \cup[2 / 9,1 / 3] \cup[2 / 3,7 / 9] \cup[8 / 9,1]$, etc., where $E_{n}$ is obtained by removing the open middle third of each interval in $E_{n-1}$, so $E_{n}$ is the union of $2^{n}$ intervals, each of length $\frac{1}{3^{n}}$.

The Cantor's set is the perfect set $E=\bigcap_{n=0}^{\infty} E_{n}$. The Hausdorff dimension of the Cantor set is $s=\frac{\ln 2}{\ln 3}$ and $h^{s}(E)=1$.

If $\bar{P}$ and $\underline{P}$ are the upper and lower conditional probabilities defined as in Theorem 5 , then they satisfy the factorization property. Moreover $B$ is irrelevant to $A$ according to the definition given by Walley, but $B$ is not s-irrelevant to $A$ since condition 1s) of Definition 4 is not satisfied. The previous example shows that the notion of irrelevance and s-irrelevance are not related if $\Omega$ is an infinite set. The next theorem put in evidence this problem in a more general framework.

Theorem 6. Let $\Omega$ be a non-empty set with positive and finite Hausdorff outer measure in its dimension and let $\bar{P}$ and $\underline{P}$ be the upper and lower conditional probabilities defined as in Theorem 5. If $A$ and $B$ are two subsets of $\Omega$ such that $\operatorname{dim}_{H}(A)<\operatorname{dim}_{H}(B)<\operatorname{dim}_{H}(\Omega)$ and they have positive and finite Hausdorff outer measures in their dimensions then $B$ is irrelevant to $A$, but $B$ is not s-irrelevant to $A$.

Proof. Denote by $t, s$ and $r$ respectively the Hausdoff dimension of $A B, B$ and $\Omega$; since $\operatorname{dim}_{H}(A)<\operatorname{dim}_{H}(B)<\operatorname{dim}_{H}(\Omega)$ then we have that the Hausdorff dimension of $B^{c}$ is equal to $r$ and $t<s$. Moreover since $A$ and $B$ and their complements have positive and finite Hausdorff outer measures in their dimensions and upper conditional probability is defined as in Theorem 5 the condition $\bar{P}(A \mid B)=\bar{P}\left(A \mid B^{c}\right)=\bar{P}(A)$ becomes $\frac{h^{s}(A B)}{h^{s}(B)}=\frac{h^{r}\left(A B^{c}\right)}{h^{r}\left(B^{c}\right)}=\frac{h^{r}(A)}{h^{r}(\Omega)}$.

These equalities are satisfied since they vanish to $0=0=0$.

In the same way we can prove the equalities $\underline{P}(A \mid B)=\underline{P}\left(A \mid B^{c}\right)=\underline{P}(A)$.

Since $\operatorname{dim}_{H}(A)<\operatorname{dim}_{H}(B)$ then the event $B$ is not s-irrelevant to $A$ since condition $1 \mathrm{~s}$ ) of Definition 4 is not satisfied. $\diamond$

In the sequel the notions of s-irrelevance and s-independence are investigated in the Euclidean metric space.

Given a non-empty subset $\Omega$ of $\Re^{n}$ with Hausdorff dimension equal to $n$ and positive and finite Hausdorff outer measure in its dimension and given two subsets $A$ and $B$ of $\Omega$, we want to find conditions under which $B$ is s-relevant to $A$. Condition 1 s) of the Definition 4 
is a necessary condition for s-irrelevance and s-independence. We focus the attention on this condition with the aim to investigate s-relevance.

In (Mattila, 1984, Theorem 6.13) an important result is proven for Souslin sets that is a class of sets, which are defined in terms of unions and intersections of closed sets.

In a metric space the Souslin sets are the sets of the form

$$
E=\bigcup_{i_{1}, i_{2}, \ldots} \cap_{k=1}^{\infty} E_{i_{1}, i_{2}, \ldots, i_{k}}
$$

where $E_{i_{1}, i_{2}, \ldots, i_{k}}$ is a closed set or each finite sequence of positive integers.

Every Borelian set is a Souslin set.

Theorem 7 (Mattila, 1984). Let $\left(\Re^{n}, d\right)$ be the Euclidean metric space and let $A$ and B be two Souslin subsets of $\Re^{n}$, with $\operatorname{dim}_{H}(A)=s, \operatorname{dim}_{H}(B)=t$ and with positive Hausdorff measure in their dimension; denote by $\Psi(x, \delta)$ the closed ball with centre $x$ and radius $\delta$ and suppose that the following lower density assumption on $B$ holds

$$
\liminf _{\delta \rightarrow 0} \delta^{-t} h^{t}(B \cap \Psi(x, \delta))>0 \text { for all } x \in B .
$$

Then we have that $\operatorname{dim}_{H}(A B)=s+t-n$.

As a consequence of the previous theorem we obtain that a necessary condition for s-irrelevance between Souslin sets of $\Re^{n}$ is that the two sets and their intersection have the Hausdorff dimension equal to $n$.

Proposition 1 Let $\left(\Re^{n}, d\right)$ be the Euclidean metric space and let $A$ and $B$ be two Souslin subsets of $\Re^{n}$ with $\operatorname{dim}_{H}(A)=\mathrm{s}, \operatorname{dim}_{H}(B)=\mathrm{t}$, with positive and finite Hausdorff measure in their dimension and such that the lower density assumption on $B$ is satisfied; then $B$ is s-relevant to $A$ if $\mathrm{s} \neq \mathrm{n}$ or $\mathrm{t} \neq \mathrm{n}$.

Proof. Since $A$ and $B$ are two Souslin subsets of $\Re^{n}$ such that lower density assumption on $B$ holds then we have that $\operatorname{dim}_{H}(A B)=s+t-n$. So condition 1s) of Definition 4 is satisfied if and only if $s=t=s+t-n$, that is $s=t=n$. $\diamond$

In the next section the previous result is used to find examples of s-dependent events.

Given a non-empty subset $\Omega$ of $\Re^{n}$ with Hausdorff dimension equal to $n$ and positive and finite Hausdorff outer measure in its dimension, conditions such that $B$ is s-irrelevant to $A$ are proven. Under regular conditions such as those ones of Theorem 7 we have that condition 1s) of the Definition 4 is satisfied if and only if $\operatorname{dim}_{H}(A)=\operatorname{dim}_{H}(B)=\operatorname{dim}_{H}(A B)=n$. In the next theorem we assume that these equalities hold and sufficient conditions such that $B$ is s-irrelevant to $A$ are proven.

Theorem 8. Let $\left(\Re^{n}, d\right)$ be the Euclidean metric space and let $A$ and B be two Souslin subsets of $\Re^{n}$ with positive and finite Hausdorff measure in their dimension, such that the following lower density assumption on $B$ holds

$$
\liminf _{\delta \rightarrow 0} \delta^{-n} h^{n}(B \cap \Psi(x, \delta))>0 \text { for all } x \in B
$$

and such that $\operatorname{dim}_{H}(A)=\operatorname{dim}_{H}(B)=\operatorname{dim}_{H}(A B)=n$.

Denoted by the Hausdorff dimension of $B^{c}$ then $B$ is s-irrelevant to $A$ in the following cases:

- a) $t=n, h^{n}\left(B^{c}\right)>0$ and $h^{n}\left(B^{c}\right) h^{n}(A B)-h^{n}(B) h^{n}\left(A B^{c}\right)=0$

- b) $t=n, h^{n}\left(B^{c}\right)=0$ and $h^{n}(A)=0$ or

- $t=n, h^{n}\left(B^{c}\right)=0$ and $h^{n}(A)=h^{n}(A B)=h^{n}(\Omega)$

- c) $t<n$ and $h^{n}(A)=0$ or

- $t<n$ and $\frac{h^{t}\left(A B^{c}\right)}{h^{t}\left(B^{c}\right)}=\frac{h^{n}(A)}{h^{n}(B)}$ 
Proof. We have to prove that condition 2s) of Definition 4 is satisfied. We consider the following cases:

- a) $t=n$ and $h^{t}\left(B^{c}\right)>0$;

- b) $t=n$ and $h^{t}\left(B^{c}\right)=0$;

- c) $t<n$

In the case a) condition 2s) of the definition of s-irrelevance is $\frac{h^{n}(A B)}{h^{n}(B)}=\frac{h^{n}\left(A B^{c}\right)}{h^{n}\left(B^{c}\right)}=\frac{h^{n}(A)}{h^{n}(\Omega)}$.

Since Souslin sets are measurable with respect to every Hausdorff outer measure (Falconer, 1986, p.6) we have that $h^{n}$ is additive so that $h^{n}(A)=h^{n}(A B)+h^{n}\left(A B^{c}\right)$ and condition 2s) is satisfied if and only if $h^{n}\left(B^{c}\right) h^{n}(A B)-h^{n}(B) h^{n}\left(A B^{c}\right)=0$. In the case b) condition 2s) is $\frac{h^{n}(A B)}{h^{n}(B)}=m\left(A B^{c}\right)=\frac{h^{n}(A)}{h^{n}(\Omega)}$.

Two cases are possible $m\left(A B^{c}\right)=0$ or $m\left(A B^{c}\right)=1$.

If $m\left(A B^{c}\right)=0$ condition 2s) is satisfied if and only if $\frac{h^{n}(A)}{h^{n}(\Omega)}=0$.

If $m\left(A B^{c}\right)=1$ condition 2s) is satisfied if and only if $h^{n}(A)=h^{n}(A B)=h^{n}(\Omega)$

In the case c) two cases are possible: $0<h^{t}\left(B^{c}\right)<+\infty$ or $h^{t}\left(B^{c}\right)=0$.

If $0<h^{t}\left(B^{c}\right)<\infty$ then:

if $\operatorname{dim}_{H}\left(A B^{c}\right)<t$ then $h^{t}\left(A B^{c}\right)=0$ and condition 2s) is satisfied if and only if $h^{n}(A)=0$;

if $\operatorname{dim}_{H}\left(A B^{c}\right)=t$ and $h^{t}\left(A B^{c}\right)=0$ and condition 2s) is satisfied if and only if $h^{n}(A)=0$;

if $\operatorname{dim}_{H}\left(A B^{c}\right)=t$ and $0<h^{t}\left(A B^{c}\right)<\infty$ then $h^{n}(A)=h^{n}(A B)$ since $h^{n}\left(A B^{c}\right)=0$; so condition 2s) is saisfied if and only if $\frac{h^{t}\left(A B^{c}\right)}{h^{t}\left(B^{c}\right)}=\frac{h^{n}(A)}{h^{n}(B)}$.

If $h^{t}\left(B^{c}\right)=0$ then $h^{t}\left(A B^{c}\right)=0$ and we obtain the same condition of case $\left.\mathrm{b}\right)$. $\diamond$

Example 4 Let $\Omega=[0,1]$, let $A=[0,1]-\frac{1}{2}$ and let $B$ be the complement of the Cantor set. We are in case c) of the previous theorem so $B$ is s-irrelevant to $A$.

\section{Stochastic s-dependence for self-similar sets}

In this section some fractal sets are proven to be s-dependent showing that they do not satisfy condition 1s) of Definition 4.

Conditions under which the attractor of a finite family of similitudes and its boundary are s-dependent and two middle third Cantor sets are s-dependent are found. If coherent upper conditional probability is defined as in Theorem 5 we have that all these sets satisfy the factorization property, which is the standard definition of probabilistic dependence.

\section{$5.1 \mathrm{~s}$-Dependence of the attractor of a finite family of similitudes on its boundary}

Let $\left(\Re^{n}, d\right)$ be the Euclidean metric space. A function $f: \Re^{n} \rightarrow \Re^{n}$ is called a contraction if $d(f(x), f(y)) \leq r d(x, y)$ for all $x, y \in \Re^{n}$, where $0<r<1$ is a constant. The infimum value for which this inequality holds for all $x, y$ is called the ratio of the contraction. A contraction that trasforms every subset of $\Re^{n}$ to a geometrically similar set is called a similitude. A similitude is a composition of a dilation, a rotation and translation. A set $E$ is called invariant for a finite set of contractions $\left\{f_{1}, f_{2}, \ldots, f_{m}\right\}$ if $E=\bigcup_{i=1}^{m} f_{i}(E)$.

If the contractions are similitudes and for some $s$ we have $h^{s}(E)>0$ but $h^{s}\left(f_{i}(E) \cap f_{j}(E)\right)=0$ for $i \neq j$ then $E$ is self similar. For any finite set of contractions there exists a unique non-empty compact invariant set $K$ (Falconer, 1986, Theorem 8.3), called attractor.

Given a finite set of contractions $\left\{f_{1}, f_{2}, \ldots, f_{m}\right\}$ we say that the open set condition (OSC) holds if there exists a bounded open set $O$ such that $O \subset \bigcup_{i=1}^{m} f_{i}(O)$ and $f_{i}(O) \cap f_{j}(O)=\oslash$ for $i \neq j$. 
If $\left\{f_{1}, f_{2}, \ldots, f_{m}\right\}$ are similitudes with similarity ratios $r_{i}$ for $i=1 \ldots m$ the similarity dimension, which has the advantage of being easily calculable, is the unique positive number $s$ for which $\sum_{i=1}^{m} r_{i}^{s}=1$. If the OSC holds then the compact invariant set $K$ is self-similar and the Hausdorff dimension and the similarity dimension of $K$ are equal. If the similarity dimension is equal to $n$ then the interior of $K, K^{0}$ is non empty. In Lau (1999) it has been proven that, given a finite family of similitudes and the corresponding attractor $K$, if $K^{0}$ is non-void and Hausdorff dimension of $K$ is equal to $n$ then the Hausdorff dimension of the boundary of $K$ is less than $n$. Moreover since the lower density assumption holds for a self-similar set, from Proposition 1 of Section 4 we have that $K$ and its boundary are s-dependent. We can observe that if upper and lower probabilities are defined as in Theorem 5 then $K$ and its boundary satisfy the the factorization property.

\section{2 s-Dependence for Cantor sets}

In this subsection two middle third Cantor sets are proven to be s-dependent.

Let $([0,1], d)$ be the Euclidean metric space and let $E$ be the Cantor set. For every $x \in[0,1]$ we consider the Cantor set $x+E$ that is the the translation of $E$.

In Davis (1995) it has been proven that for every $\alpha \in[0,1]$ there exists an $x \in(0,1)$ such that the Hausdorff dimension of the intersection $x+E \cap E=(1-\alpha) \frac{\ln 2}{\ln 3}$. So for every $\alpha \in(0,1]$ we have that the two middle third Cantor sets $x+E$ and $E$ are s-dependent since condition 1s) of Definition 4 is not satisfied.

We can observe that if upper conditional probability is defined as in Theorem 5 the factorization property is satisfied and so the two middle Cantor sets are stochastically independent according to the axiomatic definition of independence.

\section{6. s-Independence for curves filling the space}

In this section the notions of s-irrelevance and s-independence for events $A$ and $B$ that are represented by curves filling the space are analyzed.In particular Peano curve, Hilbert curve and Peano-Sierpinski curve are proven to be s-independent. Curves filling the space Sagan (1994) can be defined as the limit of a Cauchy sequence of continuous functions $f_{n}$, each mapping the unit interval into the unit square. The convergence is uniform so that the limit is a continuous function, i.e. a curve. The definition of irrelevance given by Walley, which is condition 2s) of s-irrelevance, holds when the two events $A$ and $B$ are not the trivial events $(\Omega, \oslash)$. If the conditioning event $B$ is represented by a curve filling the space, we have that the complement of $B$ is the empty-set and so in this case the notion of irrelevance becomes $\bar{P}(A \mid B)=\bar{P}(A)$; and $\underline{P}(A \mid B)=\underline{P}(A)$. If $A$ and $B$ are represented by curves filling the space we obtain the following definition of s-independence.

Definition 5 Let $(\Omega, d)$ be a metric space and let $A$ and $B$ be two curves filling the space $\Omega$. Then $A$ and $B$ are s-independent if the following conditions hold

- 1s) $\operatorname{dim}_{H}(A B)=\operatorname{dim}_{H}(B)=\operatorname{dim}_{H}(A)$

- 2s) $\bar{P}(A \mid B)=\bar{P}(A)$ and $\underline{P}(A \mid B)=\underline{P}(A)$;

- 3s) $\bar{P}(B \mid A)=\bar{P}(B)$ and $\underline{P}(B \mid A)=\underline{P}(B)$;

Moreover $B$ is s-irrelevant to $A$ if conditions 1s) and 2s) are satisfied.

Theorem 9. Let $\Omega=[0,1]^{n}$ and let $\bar{P}$ and $\underline{P}$ be the upper and lower conditional probabilities defined as in Theorem 4. If $A$ and $B$ are two curves filling the space then $A$ and $B$ are s-independent. 
Proof. Since $A$ and $B$ are curves filling the space then they and their intersection have Hausdorff dimension equal to $n$. Moreover since $A$ and $B$ are measurable we have that $\bar{P}=\underline{P}=P$ and conditions 2s) and 3s) of Definition 5 become

$$
\frac{h^{n}(A B)}{h^{n}(B)}=\frac{h^{n}(A)}{h^{n}(\Omega)} \text { and } \frac{h^{n}(A B)}{h^{n}(A)}=\frac{h^{n}(B)}{h^{n}(\Omega)}
$$

that are satisfied since they vanish to $1=1 . \diamond$

As a consequence of the previous theorem we have that the Peano curve and the Hilbert curve are s-independent. Moreover if $B$ is a curve filling the space $\Omega=[0,1]^{n}$ and $A$ is any event with Hausdorff dimension equal to $n$, then $B$ is s-irrelevant to $A$.

\section{Conclusions}

In this chapter the notions of s-irrelevance and s-independence with respect to upper and lower conditional probabilities defined by Hausdorff outer and inner measures are introduced. They are used to discover probabilistic dependence for events, which are probabilistic independent with respect to the standard definition given by the factorization property or with respect to the notion of epistemic independence.

Results and examples are given for fractal sets (i.e. sets with non-integer Hausdorff dimension) which often model complex phenomena. In particular the attractor of a finite family of similitudes and its boundary are proven to be s-dependent if the open set condition holds and a sufficient condition is given such that two middle Cantor sets are s-dependent.

Moreover two curves filling the space, such as Peano curve and Hilbert curve, are proven to be s-independent.

\section{References}

P. Billingsley. (1986). Probability and measure, Wiley, USA.

G.J. Davis, Tian-You Hu. On the structure of the intersection of two middle third Cantor sets. Publications Matemàtiques, Vol. 39, 43-60, 1995.

B. de Finetti. (1970). Teoria della Probabilita', Einaudi Editore, Torino.

D. Denneberg. (1994). Non-additive measure and integral. Kluwer Academic Publishers.

S. Doria. (2007). Probabilistic independence with respect to upper and lower conditional probabilities assigned by Hausdorff outer and inner measures. International Journal of Approximate Reasoning, 46, 617-635.

L.E. Dubins. (1975). Finitely additive conditional probabilities, conglomerability and disintegrations. The Annals of Probability, Vol. 3, 89-99.

K.J. Falconer. (1986). The geometry of fractals sets. Cambridge University Press.

G. Koch. (1997). La matematica del probabile Aracne Editrice.

Ka-Sing Lau, You Xu. (1999). On the boundary of attractors with non-void interior. Proceedings of the American Mathematica Society, Vol. 128, N.6, pp.1761-1768.

P. Mattila. (1984). Hausdorff dimension and capacities of intersections of sets in $n$-space. Acta Mathematica, 152, 77-105.

C.A. Rogers. (1998). Hausdorff measures. Cambridge University Press.

H. Sagan.(1994). Space-Filling Curves. Springer-Verlag.

P. Walley. (1991). Statistical Reasoning with Imprecise Probabilities. Chapman and Hall, London. 


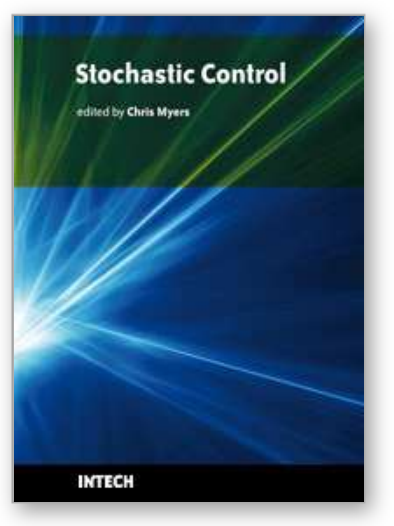

\author{
Stochastic Control \\ Edited by Chris Myers
}

ISBN 978-953-307-121-3

Hard cover, 650 pages

Publisher Sciyo

Published online 17, August, 2010

Published in print edition August, 2010

Uncertainty presents significant challenges in the reasoning about and controlling of complex dynamical systems. To address this challenge, numerous researchers are developing improved methods for stochastic analysis. This book presents a diverse collection of some of the latest research in this important area. In particular, this book gives an overview of some of the theoretical methods and tools for stochastic analysis, and it presents the applications of these methods to problems in systems theory, science, and economics.

\title{
How to reference
}

In order to correctly reference this scholarly work, feel free to copy and paste the following:

Serena Doria (2010). Stochastic Independence with Respect to Upper and Lower Conditional Probabilities Defined by Hausdorff Outer and Inner Measures, Stochastic Control, Chris Myers (Ed.), ISBN: 978-953-307121-3, InTech, Available from: http://www.intechopen.com/books/stochastic-control/stochastic-independencewith-respect-to-coherent-upper-and-lower-conditional-probabilities-de-ned-by

\section{INTECH}

open science | open minds

\section{InTech Europe}

University Campus STeP Ri

Slavka Krautzeka 83/A

51000 Rijeka, Croatia

Phone: +385 (51) 770447

Fax: +385 (51) 686166

www.intechopen.com

\section{InTech China}

Unit 405, Office Block, Hotel Equatorial Shanghai

No.65, Yan An Road (West), Shanghai, 200040, China 中国上海市延安西路65号上海国际贵都大饭店办公楼 405 单元

Phone: +86-21-62489820

Fax: $+86-21-62489821$ 
(C) 2010 The Author(s). Licensee IntechOpen. This chapter is distributed under the terms of the Creative Commons Attribution-NonCommercialShareAlike-3.0 License, which permits use, distribution and reproduction for non-commercial purposes, provided the original is properly cited and derivative works building on this content are distributed under the same license. 